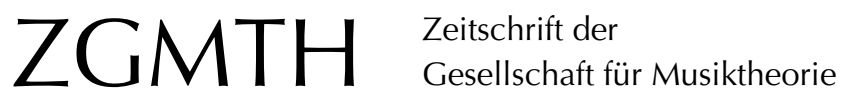

Rohringer, Stefan (2006): Die neue alte Musiktheorie. Eine Glosse. ZGMTH 3/1, 139144. https://doi.org/10.31751/211

(C) 2006 Stefan Rohringer

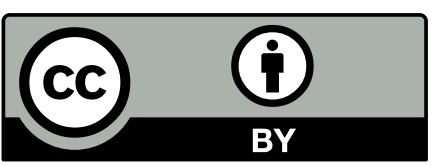

Dieser Text erscheint im Open Access und ist lizenziert unter einer Creative Commons Namensnennung 4.0 International Lizenz.

This is an open access article licensed under a

Creative Commons Attribution 4.0 International License.

veröffentlicht / first published: 01/01/2006

zuletzt geändert / last updated: 01/12/2008 


\title{
KOLUMNEN
}

\section{Die neue alte Musiktheorie}

\author{
Eine Glosse
}

\author{
Stefan Rohringer
}

Ende letzten Jahres hat der Laaber-Verlag bekanntgegeben, die Zeitschrift Musiktheorie werde in neuer Form erscheinen. Mittlerweile liegt das erste Heft des 21. Jahrgangs vor. Herausgeberteam und Layout haben gewechselt, ebenso der Titel. Er lautet nun: Musiktheorie. Zeitschrift für Musikwissenschaft. - Diese Namensgebung erstaunt.

Erinnern wir uns: Im Geleitwort der ersten Ausgabe der Musiktheorie stand zu lesen, dass sich das Periodikum als modifizierte Wiederaufnahme eines älteren Projekts begriff: Die 1970 gegründete Zeitschrift für Musiktheorie war bereits 1978 wieder eingestellt worden.

Eine deutschsprachige musiktheoretische Zeitschrift stellt ein Desiderat dar, dessen Dringlichkeit sich um so weniger bestreiten lässt, je mehr entsprechende Periodica in anderen Ländern auf reges Interesse stoßen. Dieses Interesse ist auch bei uns vorhanden. Es werden daher alle, die Musiktheorie als notwendige Ergänzung der ästhetischen Kunsterfahrung verstehen, dazu aufgerufen, Leser und Autoren der neuen Zeitschrift zu werden. ${ }^{1}$

Der seinerzeit gewählte Titel schien nicht zuletzt aus der Not geboren, den Begriff $>$ Musiktheorie als Ausweis des spezifischen Gegenstandes der Zeitschrift zu erhalten, auf die passende Bezeichnung des Organs als Zeitschrift für Musiktheorie aber nicht mehr zurückgreifen zu können.

Nun könnte man es bei der Vorstellung belassen, die neue Namensgebung sei ebenfalls aus der Not entstanden: auch eine Zeitschrift für Musikwissenschaft hat es schon gegeben. Doch gereicht die Not nicht zur Tugend, und das anfängliche Amüsement über eine Stilblüte verkehrt sich in Ärger, wenn man nach einer Erklärung Ausschau hält. Diejenige des Verlages ist so nachdrücklich wie fragwürdig:

Mutiert die seit langen Jahren dem engsten Kreis musikwissenschaftlicher Zeitschriften zuzurechnende Zeitschrift Musiktheorie zu einer inhaltlich wie konzeptionell weit offeneren Zeitschrift für Musikwissenschaft?

1 Cahn u.a. 1986. 
lautet die rhetorisch gemeinte Frage von Henning Müller-Buscher ${ }^{2}$, und er setzt eilig fort:

Um es vorweg zu nehmen: Keinesfalls. Mit der Ausweitung des Titels Musiktheorie. Zeitschrift für Musikwissenschaft soll im neuen Zeitschriftentitel nur das nachvollzogen werden, was sich ohnehin in all den zurückliegenden Jahrgängen mehr oder weniger schon in der inhaltlichen Entwicklung andeutete.

Die beschwichtigende Botschaft lautet: Der Wechsel ist bereits vollzogen, die »Ausweitung des Titels« trägt dem Rechnung.

Die beiden neuen Herausgeber, Matthias Schmidt und Wilhelm Seidel, schließen sich der Argumentation ihres Verlegers an. Ihnen zufolge hat sich die Musiktheorie vom "Spezialorgan« der frühen Jahre, mit »Themen aus dem Bereich der Musiktheorie im engeren Sinne, Beiträge[n] zur Theorie und kompositorischen Praxis des Tonsatzes, des Rhythmus und der Form« zu einer Publikation entwickelt, die zunehmend durch »ästhetisch und analytisch orientierte Studien, musikalische Kunststudien sowie Arbeiten über die Geschichte des Faches und seine Methoden« geprägt worden sei: „Die Autoren realisierten damit mehr und mehr die Musiktheorie in ihrem weiten, allgemeinen Sinne. $\aleph^{3}$ Abschließend heißt es:

Verlag und Herausgeber haben sich entschlossen, aus diesem Faktum die Konsequenz zu ziehen. Sie versehen mit dem vorliegenden Heft die Musiktheorie mit dem Untertitel Zeitschrift für Musikwissenschaft, um den Titel des Organs mit seinem Inhalt in Einklang zu bringen. ${ }^{4}$

Die Passage hat einen eigentümlichen Klang - so, als unterläge die Entwicklung der Zeitschrift einer geschichtlichen Notwendigkeit. Das Verb »realisieren« verweist nicht allein darauf, was die Autoren mit ihren Beiträgen verwirklicht haben, ihm eignet die Konnotation eines Bewusstseinswandels. Darauf wird an zentraler Stelle dieses Beitrags zurückzukommen sein. Wie sweit` es nunmehr zugehen soll, verrät wiederum MüllerBuscher:

'Musiktheorie versteht sich als umfassender Begriff, der die Identifikation mit den Wissens- und Forschungsgegenständen der 'Wissenschaft von der Musikı sucht, gar sie selbst repräsentiert. ${ }^{5}$

Diese Formulierung ist freilich recht enigmatisch, denn der Begriff Musiktheorie vermag von sich aus nichts zu suchen, schon gar keine Identifikation. Die rhetorische Figur entschuldigt nicht den Euphemismus, der mit der Anthropomorphisierung einhergeht: Begriffe sind nicht selbsttätig. `Musiktheorie` als repräsentativen Begriff für eine `Wissen-

2 Müller-Buscher 2006.

3 Schmidt/Seidel 2006.

4 Ebd.

5 Müller-Buscher 2006, 2. 
schaft von der Musikı zu verstehen bedeutet, den Begriff der `Theorie` im generellen der 'Wissenschaftı aufgehen zu lassen. sMusiktheorie Synonymen. Ein diskreter Begriff von ^Musiktheorie` wird zum Verschwinden gebracht.

So ist denn die neue Titelgebung keine Präzisierung, sondern eine Tautologie. Die Zeitung heißt eigentlich: »Musikwissenschaft. Zeitschrift für Musikwissenschaft« - mehr noch: »Musikwissenschaft. Zeitschrift für die Musikwissenschaft». In ihr soll sich »die Disziplin Musikwissenschaft in voller Bandbreite wieder finden, soll mitten im Zentrum musikwissenschaftlicher Forschung installiert werden $\ll^{6}$ und "als sSprachrohr der Musikwissenschaft intensiv in das Zentrum des Faches integriert " ${ }^{7}$ sein. Doch das Szenario zeigt Risse. Wie bereits zitiert, ist auch im Vorwort der beiden neuen Herausgeber von der "Geschichte des Faches und seinen Methoden « die Rede - nur mit dem Unterschied, dass dort der Zusammenhang darauf schließen lässt, dieses Fach heiße >Musiktheorie und nicht (historische) `Musikwissenschaft‘. Und tatsächlich: Wie die beiden neuen Herausgeber treffend bemerken, wird »eine Zeitschrift [...] nicht zuletzt durch ihre Autoren geprägt «. ${ }^{8}$ Wer aber Autor wurde, das entschieden auch bei der Musiktheorie von jeher zuallererst die jeweiligen Herausgeber. Das anfängliche Team ${ }^{9}$ bestand wie das neue ${ }^{10}$ ausschließlich aus Vertretern der historischen Musikwissenschaft. In den vergangenen Jahren allerdings agierten zunächst mit Clemens Kühn und später mit Dieter Torkewitz zwei Herausgeber, die ungeachtet ihrer musikwissenschaftlichen Promotion herausragende Vertreter des Faches Musiktheorie sind. Insbesondere dem Engagement von Clemens Kühn ist es zu danken, dass zunehmend auch solche Autoren in der $\mathrm{Mu}$ siktheorie zu Wort kamen, die nicht der historischen Musikwissenschaft zuzurechnen sind, sondern ihre Aufgaben in den Bereichen Musiktheorie, Tonsatz oder Gehörbildung an Musikhochschulen wahrnehmen. Diese Entwicklung vermochte kein entscheidendes Gegengewicht zur Tendenz der ausgewiesen musikwissenschaftlichen Kollegen zu bilden, die ursprüngliche Akzentuierung der Zeitschrift aufzugeben. Möglicherweise war sie von Verlagsseite sogar unerwünscht.

Dass die Musiktheorie in einer Zeitschrift, die diesen Namen trägt, zunehmend verwaist, bedarf der genaueren Erklärung. Man wird sie im veränderten Stellenwert musiktheoretischer Themen in der Musikwissenschaft zu suchen haben. Die Musiktheorie sah sich bei ihrer Gründung der ersten Herausgebergeneration zufolge zwei Grundsätzen verpflichtet: Zum einen sollten

6 Ebd.

7 Ebd.

8 Schmidt/Seidel 2006, 1.

9 Die Herausgeber ab dem Gründungsjahr 1986 waren zunächst Peter Cahn, Hermann Danuser, Renate Groth und Giselher Schubert, ab 1997 waren es Rudolf Bockholdt, Peter Cahn, Anselm Gerhard und Clemens Kühn, ab 2003 folgte auf Anselm Gerhard Dieter Torkewitz. Das letzte Heft des 20. Jahrgangs bestritten nur noch Peter Cahn, Clemens Kühn und Dieter Torkewitz.

10 Es ist zudem um einen Beirat ergänzt, der ebenfalls - mit einer Ausnahme, welche die systematische Musikwissenschaft betrifft - allein aus Vertretern der historischen Musikwissenschaft zusammengesetzt ist. Ihm gehören an: Wolfgang Horn, Oliver Huck, Sebastian Klotz, Annette Kreutziger-Herr, Birgit Lodes, Hartmut Möller und Wolfram Steinbeck. 
zum Gegenstandsbereich ihrer Themen [...] alle Probleme zählen, die je unter Musiktheorie diskutiert und abgehandelt wurden, und zwar ungeachtet der Frage, ob ein Problem noch heute unter sie gefasst werde oder nicht. «11

Mehr noch aber sollte die Musiktheorie von einem zweiten Grundsatz mit »stärkerem Gegenwarts- und Aktualitätsbezug« geleitet sein. Die entscheidende Passage hierzu lautet:

Das historische Interesse wird nämlich ergänzt durch ein systematisches - zum Teil auch dort, wo es darum geht, geschichtliche Fragen der Musiktheorie zu klären. Dieses systematische Interesse, das sich von Denkformen der Gegenwart herleitet und um die begrenzte Geltung möglicher Systematisierungsversuche weiß, sucht einem spezifischen Aktualitätsbedürfnis Rechnung zu tragen als die (ohnehin kaum mögliche) Klärung der Frage, wie es seigentlich gewesen sei. Es sollen also ältere musiktheoretische Positionen nicht allein sgedeutetı, sondern, wo möglich, und wünschenswert, auch sweiterentwickelt` werden. Es sollen aber auch neue wissenschaftliche Ansätze und Begriffsbildungen thematisiert und vorgestellt werden, welche die musiktheoretische Reflexion fördern, seien diese nun bezogen auf die Musik der Gegenwart oder auf ältere Musik. ${ }^{2}$

Rückblickend darf man sagen: Keine dieser beiden Hoffnungen hat sich nachhaltig erfüllt. Und insbesondere von der zweiten steht zu vermuten, dass sie sich überhaupt nicht erfüllen konnte. Zu stark war das historische Paradigma der Disziplin, die sich als systematische versuchen wollte. Obwohl mit der Formulierung von dem »systematischen Interesse, das sich von Denkformen der Gegenwart herleitet und um die begrenzte Geltung möglicher Systematisierungsversuche weiß « eine treffliche Formel gefunden war, die neuerliche Grundlegung einer systematischen Musiktheorie zu legitimieren, trat die gewünschte Weiterentwicklung nicht ein. Das "spezifischere Aktualitätsbewusstsein« der deutschen Musikwissenschaft war falsch eingeschätzt worden. Übersehen wurde, dass schon zur Zeit der Gründung der Musiktheorie in der Musikwissenschaft - mit Ausnahme der propädeutischen Form einer Allgemeinen Musiklehre - Musiktheorie fast ausschließlich als `Geschichte der Musiktheorie` begegnete. ${ }^{13}$ Die bei Grundlegung der akademischen Musikwissenschaft am Ausgang des 19. Jahrhunderts noch als »spekulative Musiktheorie« unter der systematischen Musikwissenschaft mit aufgeführte Disziplin war schon wenig später durch das Fallbeil des Historismus zu Tode gekommen. Der Wiederaufbau der deutschen Musikwissenschaft nach 1945 stellt die äußere Zäsur dar, an der der Wandel in Intention und Struktur abgelesen werden kann. Die Erkenntnis, dass Musik, und folglich alle Theorie über sie, rein geschichtlicher Natur sind, hat in der Musiktheorie zu einem tiefgreifenden Umwandlungsprozess geführt. Einer gängigen Einschätzung zufolge besteht die Crux der Musiktheorie darin, dass sie den Verdacht, nicht

11 Cahn u.a. 1986, 2.

12 Ebd., 3.

13 In dieser Hinsicht traf sich die Intention mit dem Projekt des staatlichen Instituts für Musikforschung in Berlin unter Friedrich Zaminer, eine auf 15 Bände angelegte Geschichte der Musiktheorie herauszugeben, deren erster Band 1984 erschienen war. 
allen Formen überzeitlicher Begründungssuche entsagt zu haben, niemals zur Gänze abschütteln konnte. Das aber reicht an den Kern des Problems nicht heran. Entscheidend ist, dass auch die `Arbeit am Begriffı, welche einer jeden (nicht nur musiktheoretischen) Systematik inhärent ist, und die in bester hermeneutischer Absicht historische Gegenstände neu zu durchdringen sucht, einer historischen Wissenschaft wie der historischen Musikwissenschaft notwendig zur >Geschichte der Irrtümer (Michael Polth) gerinnt. Der historische Musikwissenschaftler stellt die theoretischen Entwürfe in Vergangenheit und Gegenwart dar, er hinterfragt, auf welche Probleme die jeweiligen Theorien eine Antwort zu geben suchen, oder benennt die neuen Probleme, die aus ihnen erwachsen. Nur eines betreibt er mit Sicherheit nicht: Theoriebildung im emphatischen Sinn. Zu dieser trägt er allenfalls mittels Kritik indirekt bei. ${ }^{14}$

Das hier aufscheinende Problem ist also ein viel grundsätzlicheres, eines, für das die fragwürdige Titelgebung des Laaber-Verlages allenfalls ein Symptom bildet. Das »spezifischere Aktualitätsbewusstsein « - das darf gesagt werden, auch wenn dieser Zusammenhang seinerzeit von den Herausgebern so nicht explizit hergestellt worden ist - ist ohne die Überzeugung, »Musiktheorie [sei] notwendige Ergänzung der ästhetischen Kunsterfahrung«, nicht denkbar. An dieser Stelle wird der Unterschied zwischen einer `Kunstwissenschaft und einer `Geschichtswissenschaft deutlich. Die Kunstwissenschaft weiß sich abhängig von der ästhetischen Erfahrung, für die Geschichtswissenschaft hingegen ist dieser Punkt selbst dann arbiträr, wenn sie die Geschichte einer Kunst zum Gegenstand hat. Der Zusammenhang von ästhetischer Erfahrung und Kunstwissenschaft wird in der Musiktheorie dadurch geleistet, dass sie, wie eine jede systematische Musikwissenschaft, an die Voraussetzung gebunden ist, »Musik [sei] eine in bestimmter Weise kategorial geformte akustische Wahrnehmung «. ${ }^{15}$ Der Konsens aber, Musiktheorie trage zur kategorialen Formung auditiver Wahrnehmung bei, verliert in den Musikwissenschaften mehr und mehr an Rückhalt. ${ }^{16}$ Dieser Befund meint nicht das klare Bewusstsein davon, dass ein jeder musiktheoretischer Entwurf Ausdruck einer geschichtlichen Situation ist. Er bedeutet nichts weniger, als dass im Zeitalter der smodernen Kulturwissenschaften`, in dem alle kategoriale Formung in Zusammenhang mit einer umfassenden kulturellen Kontextualisierung verstanden wird, Musiktheorie - und offenbar auch die Auseinan-

14 Wie stark der Sog des historischen Paradigmas ist, zeigt sich auch in einer Publikation neueren Datums, die zumindest der Form nach jener alten Idee einer Ordnung der systematischen Musikwissenschaften dadurch Rechnung trägt, dass der Musiktheorie wieder ein Platz eingeräumt wird. Die Rede ist vom zur Zeit - ebenfalls im Laaber-Verlag - erscheinenden Handbuch der systematischen Musikwissenschaft (hg. von Helga de la Motte-Haber u.a.), das neben Bänden zur Musikästhetik, Musikpsychologie und Musiksoziologie auch einen Band zur Musiktheorie aufweist. Allerdings handelt es sich bei den Beiträgen, obwohl sie überwiegend nicht von Vertretern der historischen Musikwissenschaft verfasst worden sind, fast ausschließlich um geschichtliche Darstellungen - also wiederum um `Geschichte der Musiktheorie`. Allenfalls dort, wo neueste Entwicklungen paraphrasiert werden, rücken die Beiträge in die Nähe einer sspekulativen Musiktheorieı. Das mag nicht zuletzt der Gattung ’Handbuch geschuldet sein.

15 Dahlhaus, Carl 1982, »Musikwissenschaft und Systematische Musikwissenschaft", Kapitel II aus: Systematische Musikwissenschaft, hg. von Carl Dahlhaus u. Helga de la Motte-Haber (= Neues Handbuch der Musikwissenschaft Band 10), Laaber: Laaber, 26.

16 Als das in dieser Hinsicht einschneidende Datum kann ohne Übertreibung der Tod von Carl Dahlhaus bestimmt werden. 
dersetzung mit ihrer Geschichte - in den Musikwissenschaften nicht mehr als ein Modus der Kontextualisierung begriffen wird. Eine inhaltliche Begründung hierfür zu geben, fällt schwer, eine politische zu vermuten, liegt nahe: Musiktheoretisch dimensionierte Verstehenshorizonte gelten gemeinhin als zu abstrakt, um Bestandteil gesellschaftlich relevanter Diskurse zu werden.

Diese Einschätzung könnte durch nichts besser belegt sein als durch das erste Heft der neuen Musiktheorie. Sein Thema ist der »Kanon $« .{ }^{17}$ Kein einziger der Beiträge aber setzt sich mit der Frage auseinander, inwieweit historische oder gegenwärtige Musiktheorie an der Ausprägung eines Kanons teilhatte oder teilhat, oder auch, welchen Beitrag Musiktheorie dazu leisten kann, jenen geschichtlichen Zustand »von innen heraus als Funktionszusammenhang begreiflich $\aleph^{18}$ zu machen, der für die Konstituierung eines Kanons oder seines Verblassens mitverantwortlich ist.

Um einem möglichen Missverständnis vorzubeugen: Es geht nicht darum, wem `die Musiktheorie‘ gehört, nicht als Begriff und nicht als Zeitschrift - aus gutem Grund heißt diese Zeitschrift schlicht Zeitschrift der Gesellschaft für Musiktheorie. Die Weite des Gegenstandes `Musiktheorie`verträgt nicht nur, sie fordert divergente Herangehensweisen. Gerade deshalb aber bedarf es distinkter Begriffe. Fragwürdige Ausgrenzungen oder Vereinnahmungen sind einer solchen Unternehmung nicht dienlich. Sie lassen nur jenes »Desiderat« fühlbar werden, das die erste Herausgebergeneration der Musiktheorie zum Anlass ihrer Zeitschriftengründung nahm.

\section{Literatur}

Cahn, Peter / Hermann Danuser / Renate Groth / Giselher Schubert (1986), »Zum Geleit», Musiktheorie 1/1, 2-3.

Müller-Buscher, Henning (2006), »Zur neuen Musiktheorie. Vorwort des Verlages«, Musiktheorie. Zeitschrift für Musikwissenschaft 21/1, 2-3.

Schmidt, Matthias / Wilhelm Seidel (2006), „Zur neuen Musiktheorie, Vorwort der Herausgeber", Musiktheorie. Zeitschrift für Musikwissenschaft 21/1, 2.

Dahlhaus, Carl (1982), »Musikwissenschaft und Systematische Musikwissenschaft», in: Systematische Musikwissenschaft (= Neues Handbuch der Musikwissenschaft 10), hg. von Carl Dahlhaus und Helga de la Motte-Haber, Laaber: Laaber, 25-48.

17 Kompositions- und Satzlehre werden nicht thematisiert, es geht ausschließlich um ২Kanon` im Sinne von >Literaturkanon<.

18 Dahlhaus, Carl 1982, 29. 